\title{
A New Expression for Rhotrix
}

\author{
Abdul Mohammed \\ Department of Mathematics, Ahmadu Bello University, Zaria, Nigeria \\ Email: abdulmaths@yahoo.com \\ Received 17 March 2014; revised 17 April 2014; accepted 24 April 2014 \\ Copyright (C) 2014 by author and Scientific Research Publishing Inc. \\ This work is licensed under the Creative Commons Attribution International License (CC BY). \\ http://creativecommons.org/licenses/by/4.0/ \\ (c) (i) Open Access
}

\begin{abstract}
This paper presents a new technique for expressing rhotrices in a generalize form. The method involves using multiple array indexes as analogous to matrix expressions, unlike the earlier method in the literature, which can only be functional in a single array computational environment. The new rhotrix look will encourage the study of rhotrix algebra and analysis from a better perspective. In addition, computing efficiency and accuracy will also be improved, particularly when the operations in rhotrix space over the new expression are algorithmatized for computing machines.
\end{abstract}

\section{Keywords}

Rhotrix, Rhotrix Space, Rhotrix Generalization, Rhotrix Operations

\section{Introduction}

Rhotrix theory is a relatively new area of Mathematics, whose goal is central on representing arrays of numbers in rhomboid mathematical form, unlike matrix theory dealing with representing array of numbers in rectangular form. The concept of rhotrices of size three, also well known as base rhotrices, was introduced by Ajibade [1] as an extension of ideas on matrix-tertion and matrix-noitret, suggested by Atanassov and Shannon [2].

Expressing rhotrices of size $n$, particularly in a generalized form, has been a difficult problem to the rhotrix theorist. This may probably be the reason, why most works in rhotrix theory are communicated using rhotrices of specific size; one can see [1] [3]-[6]. Though, some attempts were made by Sani [8] and Mohammed et al. [7] to overcome such problem in literature, but the two generalizations proposed by these authors are sometimes inconvenient for presenting rhotrices as two dimensional objects.

Thus, it becomes imperative to seek for a new method of generalizing expression for rhotrices in order to allow for exactness, efficiency and convenience in presentation of research results. Furthermore, better algebra and analysis of rhotrix theory can be studied using multiple array technique as proposed in this article. 


\section{An Overview of Initial Generalized Rhotrix Expressions}

\subsection{Coupled Matrix Technique for Expressing Rhotrix in a Generalized Form}

In an attempt to find an answer to the problem of "finding a transformation of rhotrix to matrix and vice versa" posed by Ajibade [1] in the concluding remark of his article, Sani [8] proposed an alternative method for multiplication of rhotrices of size 3 , based on their rows and columns vectors, as comparable to matrices, recorded as follows:

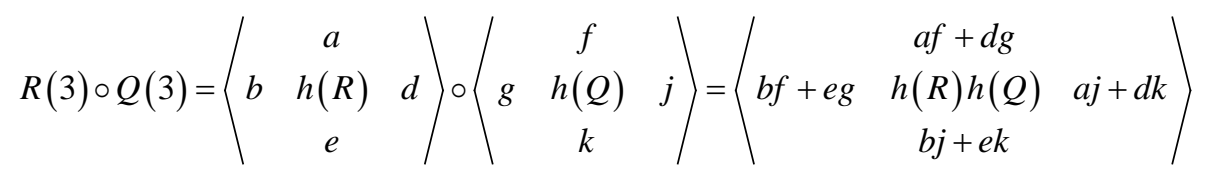

This alternative multiplication approach was also used in his work to establish some relationships between rhotrices of size 3 and $2 \times 2$ dimensional matrices through an isomorphism. Thereafter, Sani [9] extend his own method for multiplication of base rhotrices to higher size rhotrices in form of generalization, recorded as follows:

$$
R(n) \circ Q(n)=\left\langle a_{i 1 j 1}, c_{l 1 k 1}\right\rangle \circ\left\langle b_{i 2 j 2}, d_{l 2 k 2}\right\rangle=\left\langle\sum_{i 2 j 1=1}^{m}\left(a_{i 1 j 1} b_{i 2 j 2}\right), \sum_{i 2 k 1=1}^{m-1}\left(c_{l 1 k 1}, d_{l 2 k 2}\right)\right\rangle,
$$

where $R(n)=\left(\begin{array}{ccccccc} & & & a_{11} & & & \\ & & a_{21} & c_{11} & a_{12} & & \\ & \cdots & \cdots & \cdots & \cdots & \cdots & \\ a_{m 1} & \cdots & \cdots & \cdots & \cdots & \cdots & a_{m m} \\ & \cdots & \cdots & \cdots & \cdots & \cdots & \\ & & a_{m m-1} & c_{m-1 m-1} & a_{m-1 m} & & \end{array}\right)$,

$a_{i j}$ and $c_{k l}$ represent the $a_{i j}$ and $c_{k l}$ elements respectively, $m=(n+1) / 2$ with $i, j=1,2,3, \cdots, m$ and $l, k=1,2,3, \cdots, m-1$. This idea of expressing rhotrix of size $n, n \in 2 Z^{+}+1$, as a combination of two matrices, was later presented as an idea for conversion of a rhotrix to a special form of a matrix, termed "coupled matrix" in Sani [10]. That is, a rhotrix $R$ of size $n$ can be expressed as a couple of two matrices $A$ and $C$ of sizes $m \times m$ and $(m-1) \times(m-1)$ respectively, where $m=(n+1) / 2$ and $n \in 2 Z^{+}+1$.

It is noteworthy to mention that, this method presented by Sani for expressing rhotrix in a generalised form, requires some form of transformation or half transpose, in order to identify the rows and columns for any given rhotrix. Also, unique expression for the "rhotrix heart" cannot be deduced and therefore, this method of rhotrix expression is unsuitable for presenting heart-based rhotrices.

\subsection{Single Array Technique for Expressing Rhotrix in a Generalized Form}

Mohammed et al. [7] employ a single dimensional array routine for expressing real rhotrices of size $n$ in a generalised form, recorded as follows:

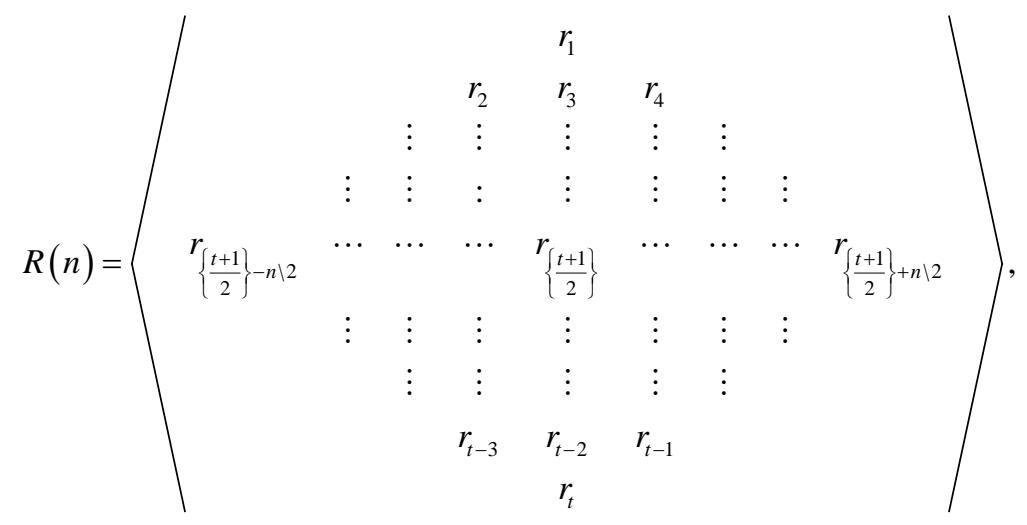


where $t=\frac{1}{2}\left(n^{2}+1\right), n \in 2 Z^{+}+1$ and $n \backslash 2$ is the integer value from division of $n$ by 2 . The entry $h(R)=r_{\frac{t+1}{2}}$ is called the heart of any rhotrix $R(n)$. The operations of addition, scalar multiplication and multiplication (o) with respect to this generalization were discussed in [7].

At this point, it is necessary to point out that this method of expressing rhotrices in generalized form follow the initial definition given for rhotrices in [1] but it can only be functional on computing machines using single array procedure, which is inadequate and capable of causing slow processing of operations involving rhotrices having the same bigger size. Also, entries in rhotrices cannot be immediately indicated.

\section{A New Generalized Expression for Rhotrix}

Since rhotrix theory is a relatively new paradigm of matrix theory, finding a generalized expression that precisely represents rhotrices of the same size $n$, while preserving row and column entries, cannot be divorced from multiple array indexes as obtainable in matrices. For this reason, an adoption of the procedure that is analogous to representation of matrices will be utilized.

For example, consider a zero heart rhotrix $A$ of size $n=5$ given below;

$$
A(5)=\left\langle\begin{array}{ccccc} 
& & 1 & & \\
& 4 & 3 & 8 & \\
10 & -7 & 0 & -6 & 5 \\
& -4 & 7 & 9 &
\end{array}\right\rangle .
$$

Clearly, if we use comparison with matrix row and column entries then by observation, the entry value 1 in rhotrix $A$ can be found at row 1 and column 3; the entry value 8 in rhotrix $A$ can be found at row 2 and column 4; the entry value- 4 in rhotrix $A$ can be located at row 4 and column 2; the entry value 0 in rhotrix $A$ can be located at row 3 and column 3; and the entry value 8 in rhotrix $A$ can be found at row 5 and column 3 . Thus, we can adopt this procedure to give a new expression for the rhotrix $R(n)$.

Let $\stackrel{\ominus}{C}(n)$ be the set of all rhotrices of size $n$ over a field $F$ (real or complex). Since any rhotrix $B(n) \in \stackrel{\ominus}{C}$ has total number of entries as $\frac{1}{2}\left(n^{2}+1\right)$, where $n \in 2 Z^{+}+1$ then a technique for expressing the set of all rhotrices of the same size $n$ in a generalized form is given by

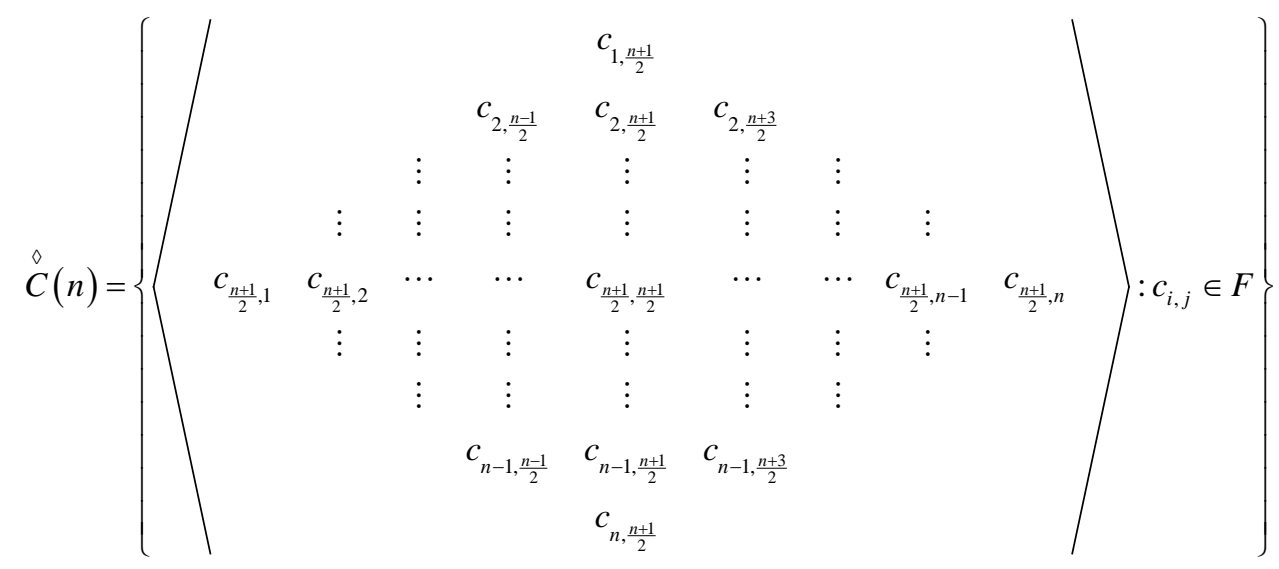

where, $c_{i j}$ are entries from a field of real or complex numbers $F$. It is clear that any entry $b_{i j}$ in $B(n)$ must be indicated by $i$ th row and $j$ th column. This method of expressing rhotrices in a general form is analogous to that of matrices because any entry $c_{i j}$ within a rhotrix is indicated out by its row $i$ and column $j$, which is analogous to any entry $x_{i j}$ in matrix set $X$ of all $m \times n$ dimensional matrices, denoted by 


$$
X(n, m)=\left\{\left[\begin{array}{cccc}
x_{11} & x_{12} & \cdots & x_{1 n} \\
x_{21} & x_{22} & \cdots & x_{2 n} \\
\vdots & \vdots & \ddots & \vdots \\
x_{m 1} & x_{m 2} & \cdots & x_{m n}
\end{array}\right]: x_{11}, x_{12}, \cdots, x_{m n} \in F\right\}
$$

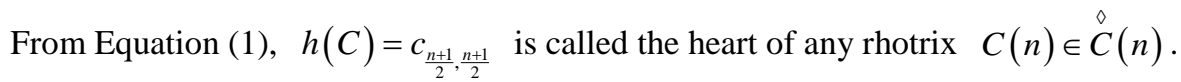

Thus, for $n=3$, we get $C(3)=\left\langle\begin{array}{ccc} & c_{11} & \\ c_{21} & c_{22} & c_{23} \\ & c_{32}\end{array}\right\rangle$ and for $n=5$, we get

$$
C(5)=\left\langle\begin{array}{lllll} 
& & c_{13} & & \\
& c_{22} & c_{23} & c_{24} & \\
c_{31} & c_{32} & c_{33} & c_{34} & c_{35} \\
& c_{42} & c_{43} & c_{44} &
\end{array}\right.
$$

\section{Operations in Rhotrix Space over the New Expression}

Let $R$ and $S$ be two rhotrices of the same size $n$ and let $\alpha$ be a scalar. If we denote the entries in rhotrix $R$ as $r_{i j}$ and the entries in rhotrix $S$ as $s_{i j}$, then we can extend the operations of addition, scalar multiplication and multiplication (o) defined for heart-base rhotrices of size-3 in [1] to rhotrices of the same $n$-size, using the rhotrix new expression respectively as follows:

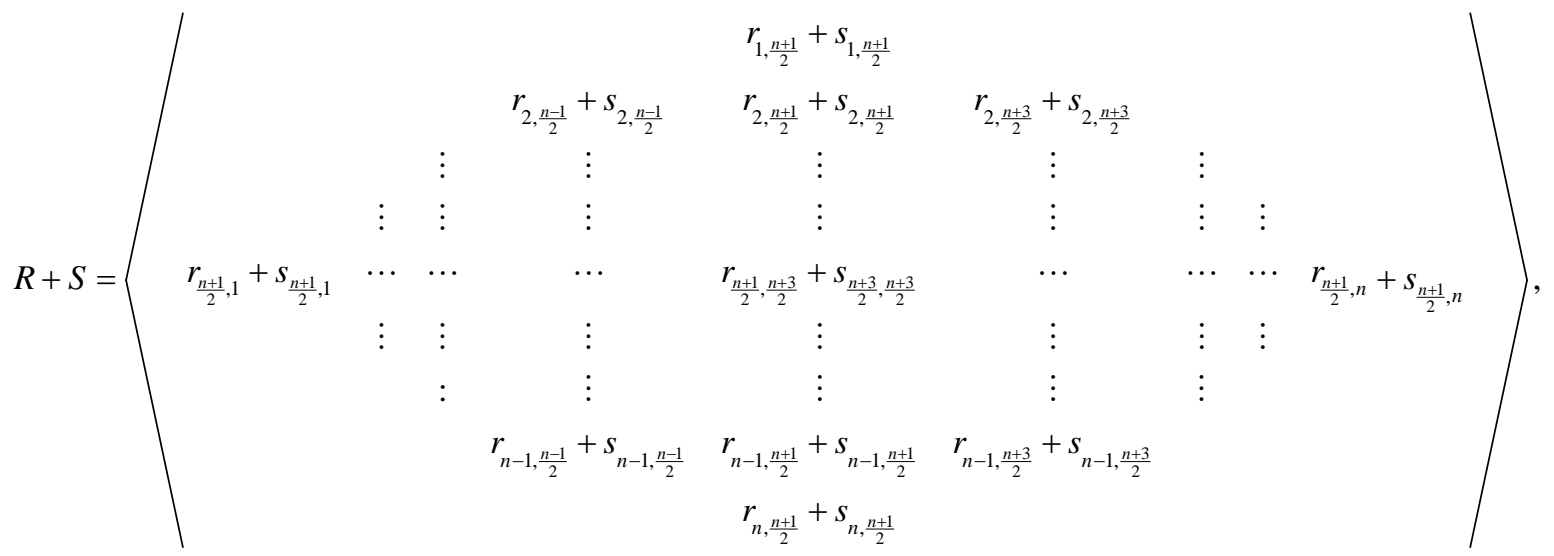

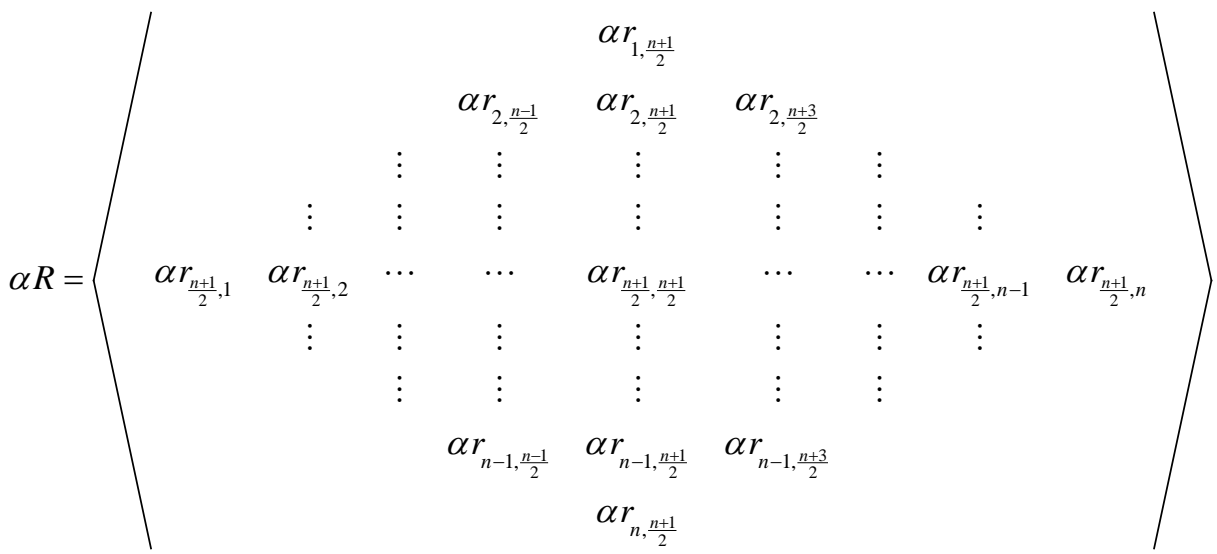

and 


$$
R \circ S=Q=\left(\begin{array}{ccccccccc} 
& \multicolumn{10}{|c}{q_{1, \frac{n+1}{2}}} & & & & \\
& & q_{2, \frac{n-1}{2}} & q_{2, \frac{n+1}{2}} & q_{2, \frac{n+3}{2}} & & & \\
& \vdots & \vdots & \vdots & \vdots & \vdots & & \\
& \vdots & \vdots & \vdots & \vdots & \vdots & \vdots & \vdots & \\
q_{\frac{n+1}{2}, 1} & q_{\frac{n+1}{2}, 2} & \cdots & \cdots & q_{\frac{n+1}{2}, \frac{n+1}{2}} & \cdots & \cdots & q_{\frac{n+1}{2}, n-1} & q_{\frac{n+1}{2}, n} \\
& \vdots & \vdots & \vdots & \vdots & \vdots & \vdots & \vdots & \\
& & \vdots & \vdots & \vdots & \vdots & &
\end{array}\right.
$$

where,

$$
\begin{gathered}
h(R)=r_{\frac{n+1}{2}, \frac{n+1}{2}} ; h(S)=s_{\frac{n+1}{2}, \frac{n+1}{2}} \\
q_{1, \frac{n+1}{2}}=r_{1, \frac{n+1}{2}} h(S)+s_{1, \frac{n+1}{2}} h(R), \\
q_{2, \frac{n-1}{2}}=r_{2, \frac{n-1}{2}} h(S)+s_{2, \frac{n-1}{2}} h(R), \\
q_{2, \frac{n+1}{2}}=r_{2, \frac{n+1}{2}} h(S)+s_{2, \frac{n+1}{2}} h(R), \\
q_{2, \frac{n+3}{2}}=r_{2, \frac{n+3}{2}} h(S)+s_{2, \frac{n+3}{2}} h(R), \\
q_{\frac{n+1}{2}, 1}=r_{\frac{n+1}{2}, 1} h(S)+s_{\frac{n+1}{2}, 1} h(R), \\
q_{\frac{n+1}{2}, 2}=r_{\frac{n+1}{2}, 2} h(S)+s_{\frac{n+1}{2}, 2} h(R), \\
q_{\frac{n+1}{2}, \frac{n+1}{2}}=r_{\frac{n+1}{2}, \frac{n+1}{2}} h(S)+s_{\frac{n+1}{2}, \frac{n+1}{2}} h(R), \\
q_{\frac{n+1}{2}, n-1}=r_{\frac{n+1}{2}, n-1} h(S)+s_{\frac{n+1}{2}, n-1} h(R), \\
q_{\frac{n+1}{2}, n}=r_{\frac{n+1}{2}, n} h(S)+s_{\frac{n+1}{2}, n} h(R), \\
q_{n-1, \frac{n-1}{2}}=r_{n-1, \frac{n-1}{2}} h(S)+s_{n-1, \frac{n-1}{2}} h(R), \\
q_{n-1, \frac{n+1}{2}}=r_{n-1, \frac{n+1}{2}} h(S)+s_{n-1, \frac{n+1}{2}} h(R), \\
q_{n-1, \frac{n+3}{2}}=r_{n-1, \frac{n+3}{2}} h(S)+s_{n-1, \frac{n+3}{2}} h(R),
\end{gathered}
$$

and $q_{n, \frac{n+1}{2}}=r_{n, \frac{n+1}{2}} h(S)+s_{n, \frac{n+1}{2}} h(R)$.

The rhotrix set $\stackrel{\ominus}{C}(n)$ together with above operations of addition $(+)$, scalar multiplication $(\alpha)$ and multiplication (०) is a generalization of rhotrix spaces, denoted by the pair $\langle\stackrel{\ominus}{C}(n),+, \circ, \alpha\rangle$.

\section{Conclusion}

We have presented a statement of expression for generalization of all rhotrices of same size- $n$ using multiple array indexes, which may be analogous to what is obtainable in matrix theory. The new rhotrix look will encourage the study of algebra and analysis of rhotrix spaces from a better perspective. In addition, computing efficiency and accuracy will also be improved, particularly when the operations over rhotrix spaces, based on the 
new expression are algorithmatized for computing machines. In the future research direction, it seems interesting to think of "axiomatization of real rhotrix space". This topic will be our next line of focus for research.

\section{Acknowledgements}

We wish to thank Ahmadu Bello University, Zaria, Nigeria for funding this relatively new area of research.

\section{References}

[1] Ajibade, A.O. (2003) The Concept of Rhotrix for Mathematical Enrichment. International Journal of Mathematical Education in Science and Technology, 34, 175-179. http://dx.doi.org/10.1080/0020739021000053828

[2] Atanassov, K.T. and Shannon, A.G. (1998) Matrix-Tertions and Matrix-Noitrets: Exercises in Mathematical Enrichment. International Journal of Mathematical Education in Science and Technology, 29, 898-903.

[3] Mohammed, A. (2007) Enrichment Exercises through Extension to Rhotrices. International Journal of Mathematical Education in Science and Technology, 38, 131-136. http://dx.doi.org/10.1080/00207390600838490

[4] Mohammed, A. (2007) A Note on Rhotrix Exponent Rule and Its Applications to Special Series and Polynomial Equations Defined over Rhotrices. Notes on Number Theory and Discrete Mathematics, 13, 1-15.

[5] Mohammed, A. (2008) Rhotrices and Their Applicationsin Enrichment of Mathematical Algebra. Proceedings of 3rd International Conference on Mathematical Sciences, United Arab Emirate University, Alain, 1, 145-154.

[6] Mohammed, A. (2009) A Remark on Classifications of Rhotrices as Abstract Structures. International Journal of Physical Sciences, 4, 496-499.

[7] Mohammed, A., Ezugwu, E.A. and Sani, B. (2011) On Generalization and Algorithmatization of Heart-Based Method for Multiplication of Rhotrices. International Journal of Computer Information Systems, 2, 46-49.

[8] Sani, B. (2004) An Alternative Method for Multiplication of Rhotrices. International Journal of Mathematical Education in Science and Technology, 35, 777-781. http://dx.doi.org/10.1080/00207390410001716577

[9] Sani, B. (2007) The Row-Column Multiplication of High Dimensional Rhotrices. International Journal of Mathematical Education in Science and Technology, 38, 657-662. http://dx.doi.org/10.1080/00207390601035245

[10] Sani, B. (2008) Conversion of a Rhotrix to a Coupled Matrix. International Journal of Mathematical Education in Science and Technology, 39, 244-249. http://dx.doi.org/10.1080/00207390701500197 